\title{
MARKERS OF OXIDATIVE STRESS AND SERUM LIPIDS IN PATIENTS WITH POLYCYSTIC OVARIAN SYNDROME
}

M. Madhu Latha, M. Vijay Bhaskar, S. S. B. Sharma, A. Sumapreethi

1. Assistant Professor, Department of Biochemistry, Mamatha Medical College and General Hospital,
Khammam.
2. Professor \& Head, Department of Biochemistry, Mamatha Medical College and General Hospital,
Khammam.
3. Associate Professor, Department of Biochemistry, Mamatha Medical College and General Hospital,
Khammam.
4. Assistant Professor, Department of Biochemistry, Mamatha Medical College and General Hospital,
Khammam.

\section{CORRESPONDING AUTHOR}

Dr. M. Madhu Latha,

Assistant Professor, Dept of Biochemistry,

Mamatha Medical College, Khammam.

E-mail: dr.madhu2m@gmail.com

Ph: 00918106890829

ABSTRACT: Dyslipidemia and oxidative stress were evaluated in patients with polycystic ovarian syndrome. MATERIALS AND METHODS: Total cholesterol, Triglyceride, HDL cholesterol, LDL cholesterol, Malondialdehyde (MDA) and Total antioxidant capacity were measured in serum of PCOS subjects and age matched controls. RESULTS: Study group comprised of 31 women with PCOS and control group with 31 healthy volunteers. Mean serum levels of MDA, Cholesterol, Triglycerides and LDL cholesterol were significantly increased and TAC and HDL cholesterol were significantly decreased in PCOS subjects compared to controls.

CONCLUSION: Our results revealed that PCOS is associated with dyslipidemia and altered oxidative status.

KEY WORDS: MDA, PCOS, TAC.

INTRODUCTION: Polycystic ovary syndrome (PCOS) is a syndrome of ovarian dysfunction that is characterized by anovulation, hyperandrogenism, and/or the presence of polycystic ovary (PCO) morphology [1].Obesity and insulin resistance occur frequently in association with this syndrome. A wide variety of risk factors have been studied in association with PCOS, including obesity, insulin resistance, dyslipidemia, endothelial dysfunction, and the presence of the metabolic syndrome $[2,3,4,5]$.

Women with PCOS would be predicted to be at high risk for dyslipidemia because they have elevated androgen levels and are frequently obese. Moreover, since they are also often hyperinsulinemic and insulin resistant, they would also be expected to be at increased risk for the dyslipidemia associated with insulin resistance. Insulin, rather than androgen, levels correlate best with lipid abnormalities, and suppressing androgen levels does not alter lipid profiles in PCOS. Insulin resistance and hyperinsulinemia are also associated with an atherogenic plasma lipid profile. Elevated plasma insulin concentrations enhance very low density lipoprotein (VLDL) synthesis, leading to hypertriglyceridemia. Progressive elimination of lipid and apolipoproteins from the VLDL particle leads to an increased formation of intermediate-density and low-density lipoproteins, both of which are atherogenic. Last, insulin, independent of its effects on blood pressure and plasma lipids, is known to be atherogenic. The hormone enhances cholesterol transport into arteriolar smooth muscle cells and increases 
endogenous lipid synthesis by these cells. Insulin also stimulates the proliferation of arteriolar smooth muscle cells, augments collagen synthesis in the vascular wall, increases the formation of and decreases the regression of lipid plaques, and stimulates the production of various growth factors ${ }^{[6,7]}$.

In summary, insulin resistance appears to be a syndrome that is associated with a clustering of metabolic disorders, including non-insulin-dependent diabetes mellitus, obesity, hypertension, lipid abnormalities, and atherosclerotic cardiovascular disease. Oxidative stress, which is generally known to be present in women with PCOS regardless of whether they are lean or have metabolic abnormalities, has been documented in infertile women[8]. And it is also reported to affect IR in these patients[9].

Oxidative stress may influence not only cardiovascular system but also female reproductive system[10].

Products of lipid peroxidation reactions have been widely employed as biomarkers for oxidative stress. MDA, produced during the decomposition of polyunsaturated fatty acids, is one of the stable end products of lipid peroxidation that can serve as a good biomarker. Various studies have measured antioxidant markers to correlate oxidative stress and PCOS and the diverse clinical manifestations of metabolic syndrome including diabetes, obesity and cardiovascular diseases. Total antioxidant capacity is the ability of serum to quench free radical production, protecting the cell structure from molecular damage. Various detection assays for TAC assay measures the combined antioxidant capacity of all its components including vitamins, proteins, lipids, glutathione, uric acid, etc[11].

The aim of our study was to investigate the relationship between PCOS, oxidative stress status and lipid profile in patients with polycystic ovary syndrome.

MATERIAL AND METHODS: In the present study, 31 PCOS patients in the age group of 21-40 years who were admitted to Gynaecology Unit of Mamata General Hospital, Khammam were recruited for the study after obtaining written informed consent (Study group). 31 healthy persons in the corresponding age group were selected from the patient's attendants and hospital staff were recruited as controls (control group).

PCOS was diagnosed according to the Rotterdam criteria[12]. The patients having two or more of the following criteria were defined as PCOS:

1. History of oligo and/or anovulation in reproductive age.

2. Clinical and/or biochemical signs of hyperandrogenism: hirsutism score of $>6$ and/or high total testosterone level.

3. Typical ovarian imaging of polycystic ovaries on ultrasound: multiple follicles in each ovary measuring 2-9 $\mathrm{mm}$ in diameter and/or increased ovarian volume $(>10 \mathrm{ml})$.

Fasting lipid profile [Total cholesterol, High density lipoprotein Cholesterol (HDLcholesterol), Triglycerides] was done to all patients by using enzymatic kits on biochemistry autoanalyser [tulip diagnostics, India]. Low density lipoprotein cholesterol values have long been estimated using the Friedwald formula:

[Total HDL cholesterol] $-20 \%$ of the Triglycerides value = Estimated LDL-cholesterol [13].

Malonaldehyde (MDA) is determined as Thiobarbituric acid reactive substances (TBARS)[14,15].

Estimation of Total Antioxidant Capacity using the FRAP (Ferric Reducing Ability of Plasma) assay.TAC assay measures the combined antioxidant capacity of all its components.(16) 
The subjects having Diabetes mellitus, Hypertension, Coronary heart disease, endocrine disorders, alcohol abuse, and on lipid lowering drugs are excluded from the study.

STATISTICAL ANALYSIS: Mean \pm S.D values of all biochemical parameters were calculated in study and control groups and the mean difference was compared by using student' $t$ ' - Test.

Mean serum cholesterol, LDL-Cholesterol, triglyceride levels were significantly increased in study group when compared to controls and mean serum HDL-Cholesterol level was significantly decreased.(TABLE 1)

These observations are consistent with previously mentioned related studies $\mathrm{T}$ Valkenburg 0 et al ${ }^{17}$, Djuro Macut et al ${ }^{18}$, Anuradha Kalra et al ${ }^{19}$.

Mean MDA level in study group was significantly increased when compared with controls. The Total antioxidant capacity in study group was significantly decreased when compared with controls (TABLE 2).

Present results are consistent with the previous studies Zhang D et al ${ }^{20}$, Palacio JR et al ${ }^{21}$ and Fenkci $\mathrm{V}$ et $\mathrm{al}^{22}$.

DISCUSSION: In our study we tried to assess lipid profile and oxidative stress in PCOS cases. We observed that mean serum levels of total cholesterol, TG, LDL-C, MDA in cases of PCOS have increased significantly, when compared with controls. Mean serum HDL-C \& TAC levels are decreased significantly when compared with controls.

Women with PCOS would be predicted to be at high risk for dyslipidemia because they have elevated androgen levels and are frequently obese. PCOS women are often hyperinsulinemic and insulin resistant, which can lead to dyslipidemia . Elevated plasma insulin concentrations enhance very-low-density lipoprotein (VLDL) synthesis, leading to hypertriglyceridemia. Progressive elimination of lipid and apolipoproteins from the VLDL particle leads to an increased formation of intermediate-density and low-density lipoproteins, both of which are atherogenic (6)

ApoA-1 - apolipoprotein A-1; ApoB - apolipoprotein B; CE - cholesteryl ester, CETP cholesteryl ester transfer protein; FFA - free fatty acid; HL - hepatic lipase; LPL - lipoprotein lipase; SD LDL, - small dense LDL cholesterol; TG - triglyceride.

Insulin resistance initiates the characteristic triad of high triglyceride level, low HDL cholesterol level and high small dense LDL level. If the concentration of VLDL-transported triglyceride is high, CETP promotes the transfer of LDL cholesteryl ester or HDL cholesteryl ester in exchange for triglyceride. Triglyceride-rich HDL cholesterol or LDL cholesterol can undergo hydrolysis by hepatic lipase or lipoprotein lipase. (22)

CONCLUSION: Dyslipidemia and increased oxidative stress is observed in patients with polycystic ovary syndrome. PCOS women should be evaluated for status of serum lipids and oxidative stress, which aids in the management of these cases. Correction of dyslipidemias and antioxidant supplementation can be beneficial in treatment of PCOS cases. It also reduces the overall morbidity and enhances the prognosis of PCOS.

ACNOWLEDGEMENT: We are extremely thankful to Dr. K. Koteshwer Rao, Dean and Principal, and Department of Obstetrics and Gynaecology, Mamatha Medical College and General Hospital, Khammam for their help.

BIBLIOGRAPH:

Journal of Evolution of Medical and Dental Sciences/Volume1/Issue5/November-2012Page-771 
1. Wild RA 2002 Long-term health consequences of PCOS. Hum Reprod Update 8:231-241]

2. Lorenz LB, Wild RA 2007 Polycystic ovarian syndrome: an evidence-based approach to evaluation and management of diabetes and cardiovascular risks for today's clinician. Clin Obstet Gynecol 50:226-243]

3. Talbott E, Guzick D, Clerici A, Berga S, Detre K, Weimer K, Kuller L 1995 Coronary heart disease risk factors in women with polycystic ovary syndrome. Arterioscler Thromb Vasc Biol 15:821-826

4. Ehrmann DA, Liljenquist DR, Kasza K, Azziz R, Legro RS, Ghazzi MN 2006 Prevalence and predictors of the metabolic syndrome in women with polycystic ovary syndrome. J Clin Endocrinol Metab 91:48-53

5. Wild S, Pierpoint T, McKeigue P, Jacobs H 2000 Cardiovascular disease in women with polycystic ovary syndrome at long-term follow-up: a retrospective cohort study. Clin Endocrinol (Oxf) 52:595-60

6. DeFronzo RA, Ferrannini E 1991 Insulin resistance . A multifaceted syndrome responsible for NIDDM, obesity, hypertension, dyslipidemia, and atherosclerotic cardiovascular disease. Diabetes Care14:173-1947.

7. Reaven GM: Banting lecture 1988: role of insulin resistance in human disease. Diabetes 37:1595-607,1988

8. Gonzalez F, Rote NS, Minium J, Kirwan JP. Reactive oxygen species-induced oxidative stress in the development of insulin resistance and hyperandrogenism in polycystic ovary syndrome. J Clin Endocrinol Metab. 2006;91:336-40.

9. Agarwal A, Gupta S, Sharma RK. Role of oxidative stress in female reproduction. Reprod Biol Endocrinol. 2005;3:28.

10. Verit FF, Erel 0. Oxidative stress in non-obese women with polycystic ovary syndrome: correlations with endocrine and screening parameters. Gynecol Obstet Invest. 2008;65:233-9.

11. Mahfouz R, Sharma R, Sharma D, Sabanegh E, Agarwal A. Diagnostic value of the total antioxidant capacity (TAC) in human seminal plasma. Fertil Steril 2009; 91(3): 805-11.

12. Rotterdam ESHRE/ASRM Sponsored PCOS Consensus Workshop Group 2004 Revised 2003 consensus on diagnostic criteria and long-term health risks related to polycystic ovary syndrome. Fertil Steril 81:19-25.

13. Friedwald WT, Levy RI, Fredrickson DS. Estimation of the concentration of low density lipoprotein cholesterol in plasma, without use of the preparative ultracentrifuge. Clin Chem. 1972; 18:499-502.

14. Sangeetha P, U.N. Das, Koratkar.R, Surya prabha.P, Increase in Free radical lipid peroxidation following chemotherapy in cancer; Free radical Biol Med 1990 ;8:15-19.

15. Iris F.F. Benzie and J.J. Strain .The Ferric Reducing Ability of Plasma (FRAP) as a Measure of "Antioxidant Power": The FRAP Assay. Analytical biochemistry 1996; 239:70-76.

16. Mahfouz R, Sharma R, Sharma D, Sabanegh E, Agarwal A. Diagnostic value of the total antioxidant capacity (TAC) in human seminal plasma. Fertil Steril 2009; 91(3): 805-11.

17. T Valkenburg 0 ,Steegers -Theunissen RP,Smedts HP,Polycystic ovary syndrome linked to atherogenic lipid profile J Clin Endocrin Metab 2007.

18. Djuro Macut, Dimitrios Panidis, Biljana Glisic, Nikolaos Spanos, Milan Petakov,Jelica Bjekic, Ivana Bozic and Svetozar Damjanovic. Relation of 
proatherogenic lipid profile and insulin resistance in PCOS Endocrine Abstracts 2008;16 P:646.

19. Anuradha Kalra, Sreekumaran Nair, Lavanya Rai . Association of obesity and insulin resistance with dyslipidemia in Indian women with polycystic ovarian syndrome Indian journal of medical sciences $2006 ; 60(11): 447-453$.

20. Zhang D, Luo WY, Liao H, Wang CF, Sun Y. The effects of oxidative Stress to PCOS. Sichuan Da Xue Xue Bao Yi Xue Ban 2008;39(3): 421-3.

21. Palacio JR, Iborra A, Ulcova-Gallova Z, Badia R, Martinez P. The presence of antibodies to oxidative modified proteins in serum from polycystic ovary syndrome patients. Clin Exp Immunol 2006; 144(2): 217-22.

22. Fenkci V, Fenkci S, Yilmazer M, Serteser M. Decreased total antioxidant status and increased oxidative stress in women with polycystic ovary syndrome may contribute to the risk of cardiovascular disease. Fertil Steril 2003; 80(1): 123-7.

23. Arshag D Mooradian. Dyslipidemia in type 2 diabetes mellitus. Nature Clinical Practice Endocrinology \& Metabolism (2009) 5, 150-159

RESULTS: TABLE 1: Mean Total Cholesterol, HDL, LDL, TG Levels In Control Group \& Study Group

\begin{tabular}{|l|l|l|l|l|l|}
\hline \multirow{2}{*}{$\begin{array}{c}\text { PARAMETERS } \\
(\mathrm{mg} / \mathrm{dl})\end{array}$} & \multicolumn{2}{|c|}{ Control group } & \multicolumn{2}{c|}{ Study group } & P value \\
\cline { 2 - 6 } & Mean & Std.Dev & Mean & Std.Dev & \\
\hline T.Cholesterol & 159.16 & 12.87 & 197.81 & 37.94 & $<0.0003$ \\
\hline HDL & 40.87 & 3.58 & 37.13 & 5.02 & $<0.0013$ \\
\hline LDL & 95.13 & 14.16 & 125.52 & 41.29 & $<0.0003$ \\
\hline Triglyceride & 113.71 & 38.36 & 146.19 & 57.77 & $<0.0115$ \\
\hline
\end{tabular}

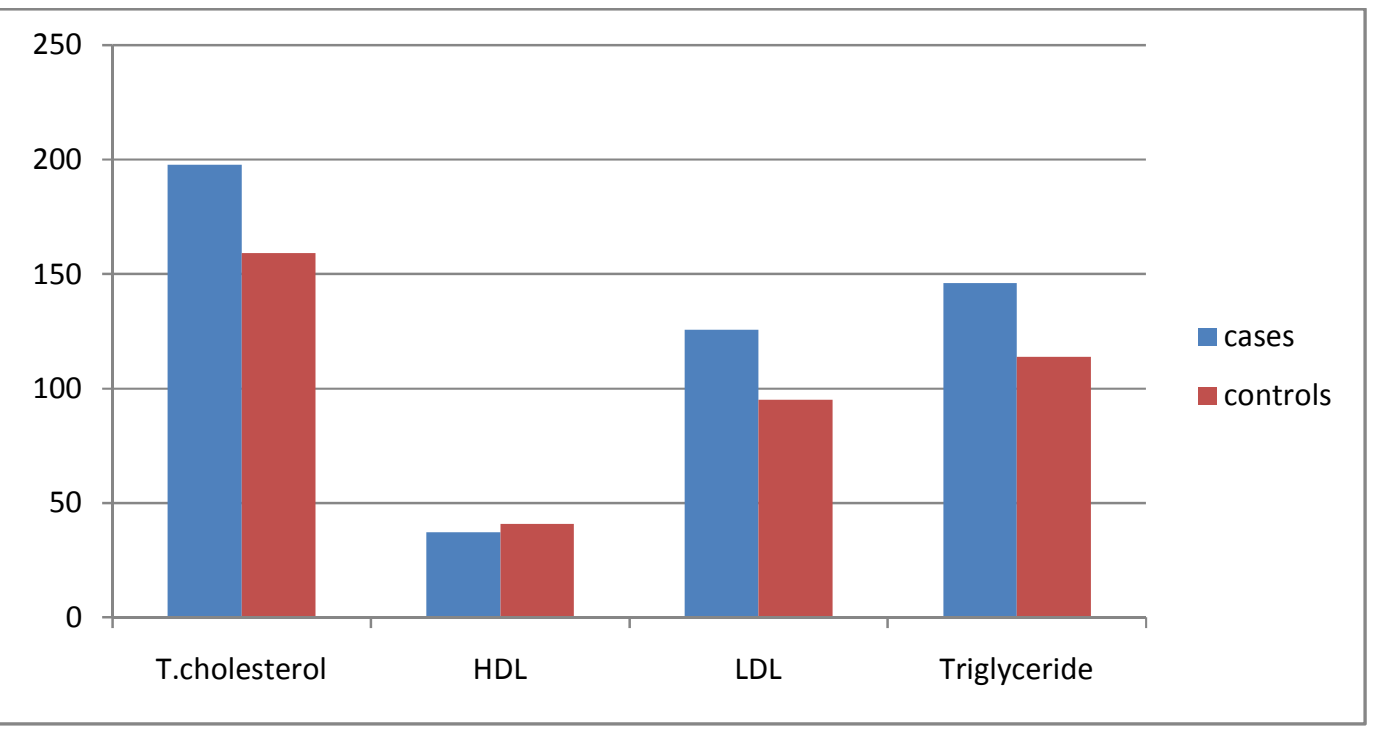

Journal of Evolution of Medical and Dental Sciences/Volume1/Issue5/November-2012Page-773 
Graph- 1

TABLE 2: Mean MDA \&TAC Levels In Control \& Study Group

\begin{tabular}{|l|l|l|l|l|l|}
\hline \multirow{2}{*}{ PARAMETERS } & \multicolumn{2}{|c|}{ Control group } & \multicolumn{2}{c|}{ Study group } & \\
\cline { 2 - 6 } & Mean & Std.Dev & Mean & Std.Dev & P value \\
\hline MDA(nmol/ml) & 1.67 & 0.53 & 3.12 & 1.16 & $<0.0001$ \\
\hline TAC(umol/ml) & 1.15 & 0.33 & 0.57 & 0.25 & $<0.0001$ \\
\hline
\end{tabular}

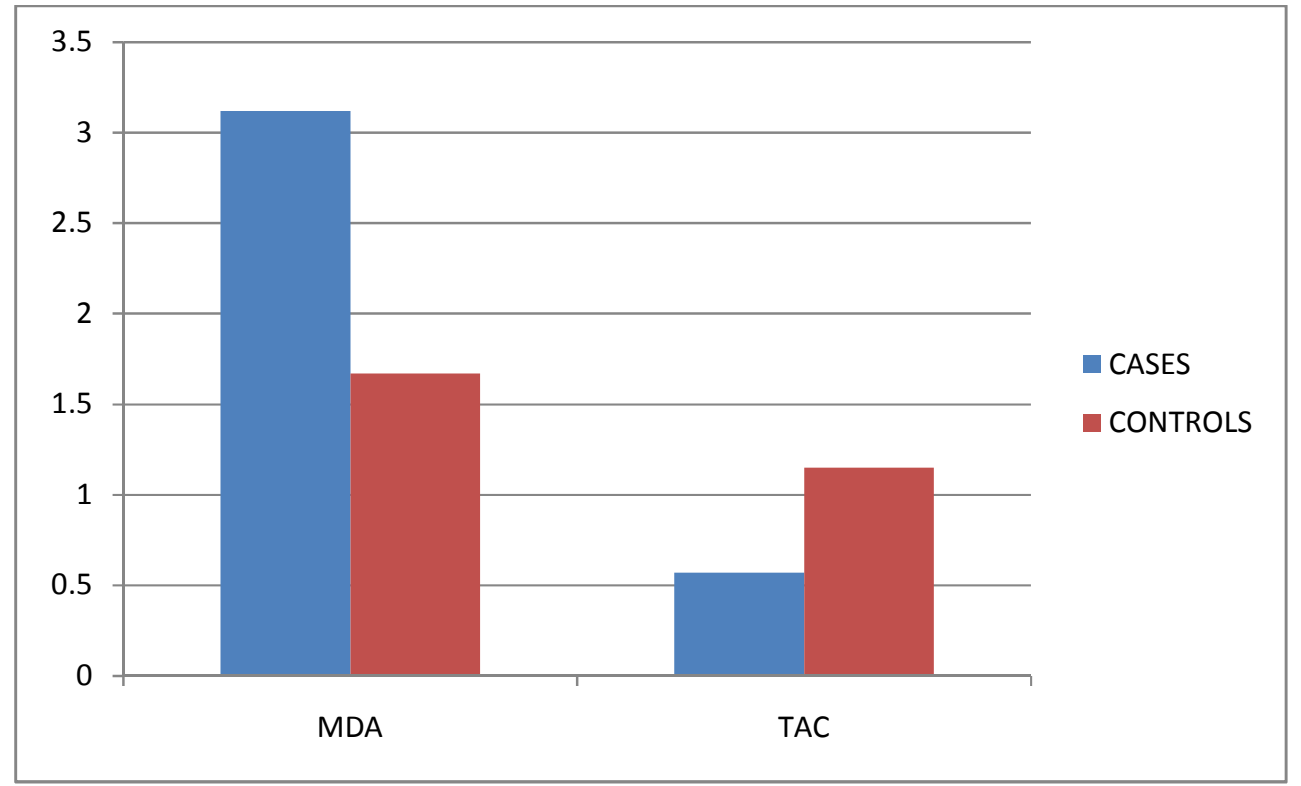

Graph -2

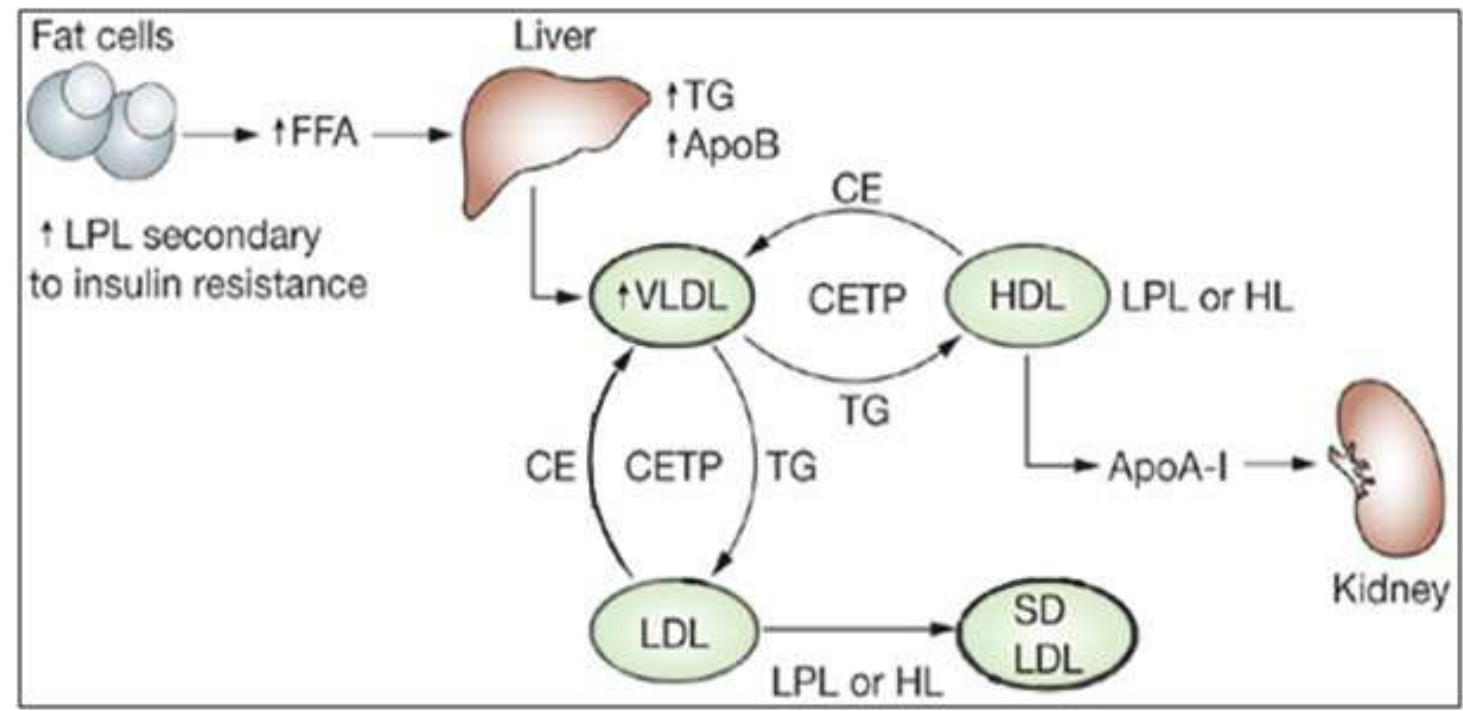

FIG.1:The role of insulin resistance in dyslipidemia 